\title{
Method for rapid multidiameter single-fiber reflectance and fluorescence spectroscopy through a fiber bundle
}

\author{
A. Amelink*a, C.L. Hoy ${ }^{\mathrm{b}}$, U.A. Gamm ${ }^{\mathrm{b}}$, H.J.C.M. Sterenborg ${ }^{\mathrm{b}}$, D.J. Robinson ${ }^{\mathrm{b}}$ \\ ${ }^{\mathrm{a}}$ TNO, Stieltjesweg 1, 2628 CK Delft, The Netherlands; ${ }^{\mathrm{b}}$ Dept. of Radiation Oncology, Erasmus \\ MC, P.O. Box 2040, 3000 CA Rotterdam, The Netherlands
}

*arjen.amelink@tno.nl.

\begin{abstract}
We have recently demonstrated a means for quantifying the absorption and scattering properties of biological tissue through multidiameter single-fiber reflectance (MDSFR) spectroscopy. These measurements can be used to correct single-fiber fluorescence (SFF) spectra for the influence of optical properties, enabling quantification of intrinsic fluorescence. In our previous work, we have used a series of pinholes to show that selective illumination and light collection using a coherent fiber bundle can simulate a single solid-core optical fiber with variable diameter for the purposes of MDSFR spectroscopy. Here, we describe the construction and validation of a clinical MDSFR/SFF spectroscopy system that avoids the limitations encountered with pinholes and free-space optics. During one measurement, the new system acquires reflectance spectra at the effective diameters of 200,600, and $1000 \mu \mathrm{m}$, and a fluorescence spectrum at an effective diameter of $1000 \mu \mathrm{m}$. From these spectra, we measure the absolute absorption coefficient, $\mu_{\mathrm{a}}$, reduced scattering coefficient, $\mu_{\mathrm{s}}$, , phase function parameter, $\gamma$, and intrinsic fluorescence, $\mathrm{Q} \mu_{\mathrm{a}}^{\mathrm{f}}$, across the measured spectrum. We validate the system using Intralipid- and polystyrene sphere-based scattering phantoms, with and without the addition of the absorber Evans Blue. Finally, we demonstrate the combined MDSFR/SFF of phantoms with varying concentrations of Intralipid and fluorescein, wherein the scattering properties are measured by MDSFR and used to correct the SFF spectrum for accurate quantification of $\mathrm{Q} \mu_{\mathrm{a}}^{\mathrm{f}}$.
\end{abstract}

Keywords: fluorescence spectroscopy; reflectance spectroscopy; scattering; tissue optics; optical property measurement.

\section{INTRODUCTION}

Optical spectroscopy of tissue is a powerful technique that provides a variety of diagnostically useful information about tissue optical properties and constituents $(1,2)$. Reflectance spectroscopy contains information about the presence and concentration of tissue chromophores, as well as ultrastructural information related to scattering (2). Meanwhile, fluorescence spectroscopy has the ability to detect endogenous molecules such as NADH and collagen as well as exogenous fluorescent markers or drugs (3). Optical spectra acquired from tissue contain the competing effects of all tissue optical properties and are also highly dependent on illumination and detection geometry. As a result, the isolation and quantitative measurement of individual tissue optical properties and tissue constituents presents a major challenge in optical spectroscopy.

Single fiber reflectance (SFR) spectroscopy, in which the illumination and detection are performed by the same optical fiber, can be used to address this challenge. In this geometry, the measurement volume is confined to shallow depths on the order of the fiber diameter, dependent on the optical properties $(4,5)$, and the measurement is sensitive to the scattering phase function (6). As such, SFR spectroscopy may be well suited for detection of localized changes to tissue microstructure that are expected to accompany early onset of disease. Additionally, the compact and simple probe design allows easy incorporation of small-diameter SFR probes into many clinical tools, such as endoscopic catheters $(7,8)$ and FNA-needles $(9,10)$

Recently, our group has shown that the tissue absorption coefficient, $\mu_{\mathrm{a}}\left[\mathrm{mm}^{-1}\right]$, can be accurately quantified without prior knowledge of the tissue scattering properties from a SFR measurement through the use of empirical models for the effective photon path-length and the collected single fiber reflectance in the absence of absorption (11). Decomposition 
of $\mu_{\mathrm{a}}$ into the constituent absorption spectra of known tissue chromophores enables accurate measurement of chromophores concentration and microvascular parameters such as local blood oxygen saturation, blood volume fraction, and mean vessel diameter, which can be used for differentiating between healthy and cancerous tissue (10). Due to the sensitivity of the SFR geometry to the scattering phase function, we have shown that acquiring at least two successive SFR measurements with different fiber diameters enables quantification of the reduced scattering coefficient, $\mu^{\prime}$ s $\left[\mathrm{mm}^{-1}\right]$, and the phase function parameter $\gamma[-]$ as well $(12,13)$. These scattering parameters are functions of the first and second Legendre moments of the scattering phase function, $g_{1}[-]$ and $\mathrm{g}_{2}[-]$, where $\gamma$ represents the likelihood of large-angle backscattering events. Specifically, an increase in $\gamma$ indicates a decrease in large-angle backscattering. The scattering phase function is directly related to the tissue refractive index correlation function through a Fourier transform relationship $(14,15)$; thus quantitative measurement of $\mu^{\prime}{ }_{s}$ and $\gamma$ can provide insight into tissue microstructure, which can be useful in diagnosing early onset of disease. The MDSFR spectroscopy technique has been validated in both Monte Carlo simulations $(12,16)$ and tissue-mimicking liquid optical phantoms $(13)$, and has recently been used in vivo to quantify optical properties in a murine cancer model (17).

The tissue optical properties measured with the multi-diameter single fiber reflectance (MDSFR) technique can be used for correction of fluorescence spectroscopy, where in situ quantification of fluorophore concentrations from fluorescence spectra is complicated by the effects of tissue optical properties on the excitation and emission light. We have recently developed a semi-empirical model for single fiber fluorescence (SFF) spectroscopy that corrects for the effects of tissue optical properties at both the excitation and emission wavelengths to enable accurate quantification of intrinsic fluorescence (18), given as the product of the tissue fluorophore absorption coefficient at the excitation wavelength, $\mu_{\mathrm{a}}{ }^{\mathrm{f}}$ $\left[\mathrm{mm}^{-1}\right]$ and the quantum efficiency across the emission spectrum, $\mathrm{Q}[-]$.

Conducting MDSFR spectroscopy by sequential placement of multiple optical fibers is time consuming and sensitive to errors in probe placement, making this approach clinically impractical. We have previously demonstrated a means of simulating a single fiber with variable diameter using a coherent fiber bundle and a series of pinholes to control the effective fiber diameter (19). While successful, this technique is still time consuming and limited by back-reflections.

Here we present the development and characterization of a MDSFR/SFF system which uses a 19-core fiber bundle and eliminates free-space optical components for improved robustness, signal-to-noise ratio, and acquisition time. In this paper, we summarize the critical aspects of the system design and the characterization and validation of the system using liquid optical phantoms.

\section{MATERIALS AND METHODS}

\subsection{Design of the MDSFR/SFF System}

The MDSFR/SFF system is based upon a custom-built fiber bundle that consists of 19 optical fibers with core sizes of $200 \mu \mathrm{m}$ (CeramOptec, Germany). At the distal end, the fibers are bundled into three concentric groups comprised of one, six, and twelve fibers, as shown in Fig. 1.
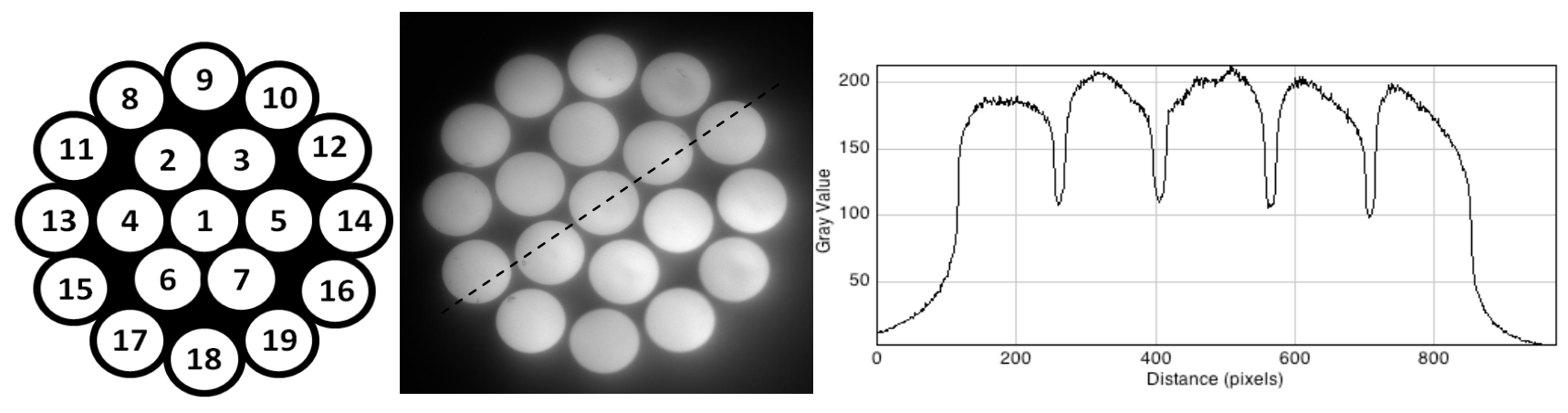

Figure 1. a) schematic representation of fiber bundle. b) image of the distal end of the fiber bundle. c) intensity distribution across the dashed line in b). 
Using a CCD camera, the outer diameter of the middle and outer rings of fiber cores were found to be $650 \mu \mathrm{m}$ and 1060 $\mu \mathrm{m}$, respectively. At the opposite end, the fibers are individually terminated to allow direct fiber optic coupling to individual cores. Each fiber in the bundle is trifurcated to connect to (1) a fiber delivering light from a halogen lamp (HL-2000-FHSA, Ocean Optics, The Netherlands), (2) a fiber delivering light from a $365 \mathrm{~nm}$ LED (NC4U133A, Nichia, Japan) or a $780 \mathrm{~nm}$ LED (L780-06-55, Marubeni, USA), and (3) a fiber collecting light returning from the sample and delivering it to one of three spectrometers. A series of fiber optic interconnects, along with three computer-controlled shutters, enables illumination and spectroscopic detection of the center fiber, the middle ring, and the outer ring of fibers, independently. Homogenous illumination of each fiber in the bundle was verified to within a standard deviation of $7.6 \%$ using a CCD camera (figure 1c).

For detection, spectra are acquired from the three groups of fibers using three spectrometers (two S2000s, one USB2000+, Ocean Optics, The Netherlands) with an overlapping spectral range of 350-1000 nm. Each spectrometer is filtered by a long pass filter with a $385 \mathrm{~nm}$ cut-off wavelength (GL-GG385-12, Avantes, The Netherlands) to remove fluorescence excitation light. For SFF, all fibers in the bundle are illuminated simultaneously by the $365 \mathrm{~nm}$ LED while detecting on all spectrometers, thus providing an SFF measurement using the largest effective fiber diameter of $1.0 \mathrm{~mm}$.

Lastly, a laptop running LabView coordinates fiber illumination, merging of the three spectrometer channels, calibration, and display of calibrated MDSFR and SFF spectra. A foot pedal is used to initiate each combined MDSFR/SFF measurement, which requires less than eight seconds to complete in the current configuration.

\subsection{System Validation}

Because the fiber bundle used in this study is more coarse that the bundle used in our previous system (7-19 fibers used to represent a single solid-core fiber, instead of 1k-10k) (19), and the effective SFR spectra are merged across multiple spectrometers, it is non-obvious whether or not the merged spectra acquired from the individual fibers are equivalent to the SFR spectra measured by a single solid-core fiber. To answer this question, we have performed a series of experiments using liquid optical phantoms to confirm that the MDSFR and SFF spectra acquired with the system are in agreement both with our models for MDSFR and SFF and with experimental MDSFR and SFF spectra acquired using single solid-core fibers of equal diameter.

First, a series of scattering and absorbing phantoms were measured by SFR using both the fiber bundle system and individual solid-core fibers with $\mathrm{d}_{\mathrm{f}}=[0.2,0.6,1.0] \mathrm{mm}$. The solid-core fiber diameters were chosen to closely match the diameters of the rings of fibers in the fiber bundle. The phantoms consisted of varying concentrations of Intralipid and the absorber Evans Blue in $0.9 \% \mathrm{NaCl}$ solution to achieve $\mu_{\mathrm{s}}{ }^{\prime}(611 \mathrm{~nm})=[0.36,0.53,0.71,1.1,1.4,1.8,3.5,5.3] \mathrm{mm}^{-1}$ (22) and $\mu_{\mathrm{a}}(611 \mathrm{~nm})=[0,0.5,1,2,3] \mathrm{mm}^{-1}$. To properly imitate a solid-core fiber for the purposes of SFR, the merged spectra from the MDSFR system must exhibit equal effective path length $<\mathrm{L}_{\mathrm{SFR}}>$ and reflectance $R_{S F}$ to that of a solidcore fiber of a given diameter across the measured spectrum. In SFR spectroscopy, the effective path length has been found to have a fixed relationship to the mean sampling depth of the measurement (4), and so demonstration of equivalent path length to single fiber measurements demonstrates that the measurement depth is also equivalent. Path length equivalence was verified by measuring $<\mathrm{L}_{\mathrm{SFR}}>$ using Eq. 1 as in previous studies $(5,19)$, where

$$
\left\langle L_{S F R}\right\rangle=-\frac{1}{\mu_{a}} \ln \left(\frac{R_{S F}}{R_{S F}^{0}}\right)
$$

Here $R_{S F}$ is the reflectance in the presence of the absorber, and $R_{S F}^{0}$ is the reflectance in the absence of the absorber. For this analysis, Eq. 1 is evaluated at $611 \mathrm{~nm}$, where the $\mu_{\mathrm{a}}$ of Evans Blue has its maximum. In addition to the path-length, $R_{S F}$ was compared between the MDSFR system and the solid-core fibers at $\lambda=[425,525,625,725,825] \mathrm{nm}$.

Lastly, a series of phantoms was prepared with varying concentrations of Intralipid in phosphate buffered saline, both with and without $20 \mu \mathrm{M}$ of fluorescein. The absorption coefficient of the fluorescein solution at $365 \mathrm{~nm}$ excitation, $\mu_{\mathrm{a}}{ }_{\mathrm{f}}^{\mathrm{f}}$, was measured with a spectrophotometer to be $7.6 \pm 0.1(10)^{-3} \mathrm{~mm}^{-1}$. MDSFR and SFF spectra were acquired from each phantom, where the $\mu_{\mathrm{a}}$ and $\mu_{\mathrm{s}}$ ' extracted from the MDSFR analysis were used to correct the SFF measurement and extract the intrinsic fluorescence. 


\section{RESULTS}

\subsection{Determination of Single Fiber Equivalence and Effective Fiber Diameter}

Analyzing the effective photon path-length in the Intralipid-based phantoms using Eq. 1, $<\mathrm{L}_{\mathrm{SFR}}>$ at $611 \mathrm{~nm}$ is observed to correlate well between the fiber bundle and the solid-core fibers with an overall Pearson correlation coefficient of $r$ $=0.987$ for the three effective diameters, as shown in Fig. $2 \mathrm{a}$. The RMS residual errors for the merged channels, $\mathrm{d}_{\mathrm{f}}=1.0$ $\mathrm{mm}$ and $0.6 \mathrm{~mm}$, of $3.93 \%$ and $5.93 \%$, respectively. The measurements for $\mathrm{d}_{\mathrm{f}}=0.2 \mathrm{~mm}$ represent comparisons between two solid-core fibers, because this channel consists of only one fiber core in the MDSFR system, and the increased scatter observed in some of these measurements arises from the reduction in signal from SFR measurements with decreasing dimensionless scattering coefficient, $\mu_{\mathrm{s}}{ }^{\prime} \mathrm{d}_{\mathrm{f}}$.
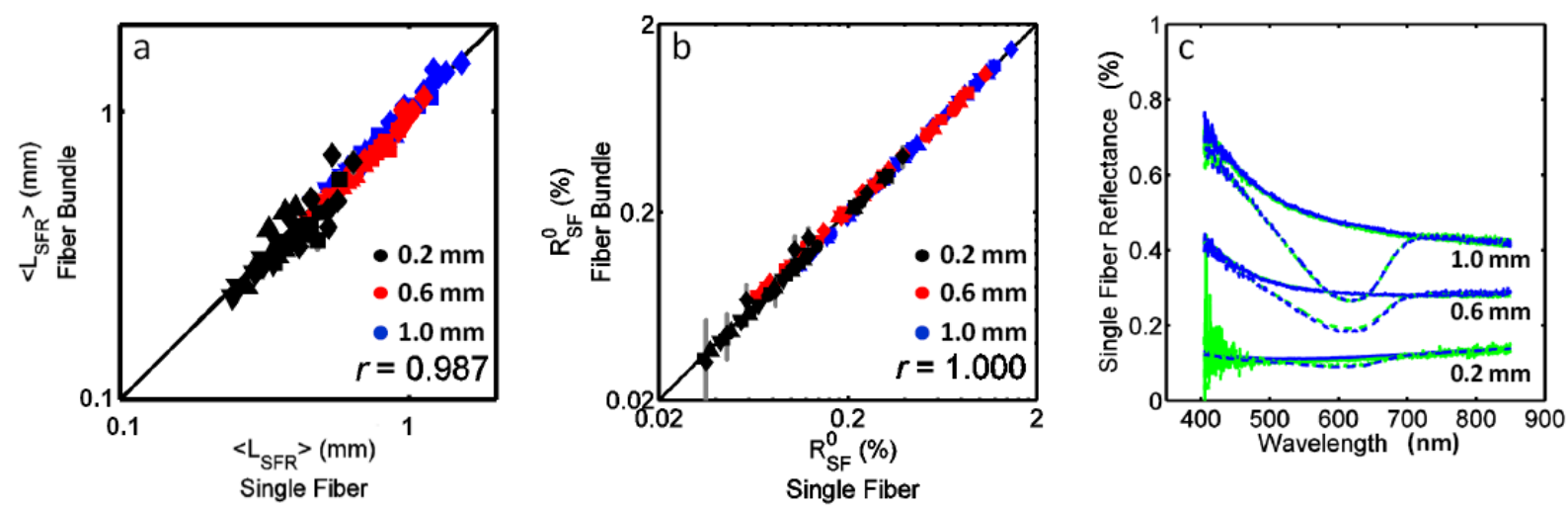

Figure 2. Comparison of SFR measurements in Intralipid phantoms between the fiber bundle system and solid-core fibers. (a) Comparison of $\left\langle\mathrm{L}_{\mathrm{SFR}}>\right.$ at $611 \mathrm{~nm}$. Data correspond to $\mu_{a}(611 \mathrm{~nm}$ ) of 0.5 (diamond), 1 (square), 2 (upright triangle), and 3 (downward triangle) $\mathrm{mm}^{-1}$, respectively. (b) Comparison of $R_{S F} 425$ (diamond), 525 (square), 625 (upright triangle), 725 (downward triangle), and 825 (circle) $\mathrm{nm}$, respectively. In (a) and (b), the blue, red, and black data correspond to fiber diameters of 1.0, 0.6, and $0.2 \mathrm{~mm}$, respectively. (c) Plot of representative SFR spectra measured with the fiber bundle system (green) and solid-core fibers (blue) for $\mu_{s}{ }^{\prime}(611 \mathrm{~nm})=1.8 \mathrm{~mm}^{-1}, \mu_{a}(611 \mathrm{~nm})=0$ and 0.5 $m m^{-1}$.

The path-length analysis above relies only on the measured reflectance at one wavelength. To determine if the reconstructed spectra in the MDSFR system are equivalent to SFR spectra from solid-core fibers across the entire spectrum, the measured for both systems were compared. Figure $2 \mathrm{~b}$ displays the correlation between the reflectance measured with the fiber bundle and measured by the solid-core fiber for a range of wavelengths. These measurements are strongly correlated $(\mathrm{r}=1.000)$ and RMS residual errors for the merged channels, $\mathrm{d}_{\mathrm{f}}=1.0 \mathrm{~mm}$ and $0.6 \mathrm{~mm}$, are $3.06 \%$ and $3.83 \%$, respectively. Figure 2c displays an overlay of representative SFR spectra measured by the fiber bundle and by the solid-core fibers.

\subsection{Validation of SFF through extraction of $Q \mu_{a}$}

The extraction of the intrinsic fluorescence of fluorescein in Intralipid scattering phantoms was used to validate quantitative fluorescence spectroscopy with the MDSFR/SFF device. In these measurements, the scattering properties of Intralipid-fluorescein phantoms were extracted from the MDSFR measurement and used to correct the SFF measurement for the effects of the optical properties. The resulting $\mathrm{Q} \mu_{\mathrm{a}}^{\mathrm{f}}$ was found to be $7.0 \pm 0.3(10)^{-3} \mathrm{~mm}^{-1}$, which yields a $\mathrm{Q}$ of $0.92 \pm 0.04$ for the measured $\mu_{\mathrm{a}}^{\mathrm{f}}$ of $7.6 \pm 0.1(10)^{-3} \mathrm{~mm}^{-1}$. The measured $\mathrm{Q}$ is in good agreement with the published value for fluorescein, $\mathrm{Q}=0.88$, at the measured $\mathrm{pH}$ of $7.4(20)$. Using the extracted $\mathrm{Q} \mu_{\mathrm{a}}^{\mathrm{f}}$ of $7.0(10)^{-3} \mathrm{~mm}^{-1}$, the dimensionless single fiber fluorescence was found to be in good agreement with the previously developed model (18) throughout the investigated range of dimensionless scattering coefficient, $\mu_{\mathrm{s}}{ }^{\prime} \mathrm{d}_{\mathrm{f}}$, as seen in Fig. 3. 

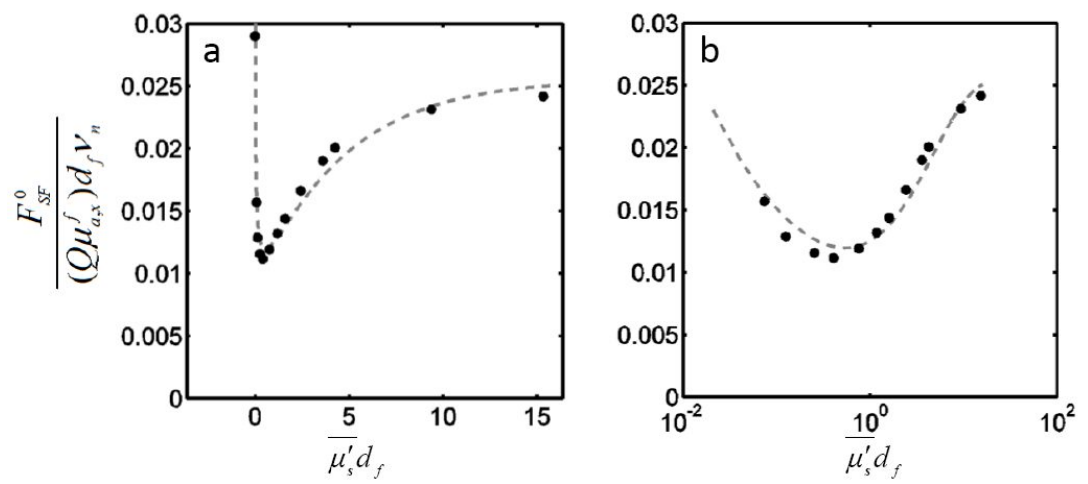

Figure 3. The nondimensionalized SFF corresponding to the best fit of $Q \mu_{a}^{f}$ to the data (black data points) and the predicted dependence on $\mu_{s}{ }^{\prime} d_{f}$ (gray dashed line) on a (a) linear scale and (b) semi-log scale.

\section{DISCUSSION}

Based on the similarity observed in Fig. 2 between the SFR spectra measured by the fiber bundle system and by the solid-core fibers, we conclude that the merged spectra from the fiber bundle system do accurately represent spectra from single solid-core fibers across the entire measured spectral range.

The fiber bundle used in this study uses only a minimum number of fibers (7 or 19) to simulate a solid-core fiber, in contrast to our previous study in which each effective fiber diameter consisted of thousands of small individual fibers. The successful simulation of solid-core fibers in both cases indicates that the coarseness of the fiber bundle has little impact on SFR measurements over the range of $\mu_{\mathrm{s}}{ }^{\prime} d_{\mathrm{f}}$ investigated. This suggests that use of a fiber bundle as a variable diameter single fiber for MDSFR is broadly applicable to a range of fiber bundle geometries between these two extreme cases. As a result, alternative fiber bundle geometries can be considered to suit specific applications. For example, a probe with $\mathrm{d}_{\mathrm{f}}=[0.2,0.4,0.6] \mathrm{mm}$ might be considered for an application wishing to focus on skin epithelial properties, while an alternative probe with $d_{f}=[0.8,1.2,1.5] \mathrm{mm}$ could be employed to increase the sensitivity to deeper chromophores (4).

The coarse bundle used in this study has several advantages for clinical use. The size and number of the individual fiber cores enables construction of the system with entirely fiber optic connections. This architecture eliminates nearly all back-reflections and provides greater efficiency in light delivery and collection, all of which improve the signal-tobackground ratio (SBR) and acquisition speed. For comparison, the previous MDSFR system based on a pinhole and free-space optics was limited by poor SBR for the $0.2 \mathrm{~mm}$ effective fiber diameter, with SBR $=0.016$ for the $\mu_{\mathrm{s}}{ }^{\prime}=3.6$ $\mathrm{mm}^{-1}$ phantom. Using the all fiber optic design presented here, the $0.2 \mathrm{~mm}$ effective diameter displayed $\mathrm{SBR}=37$ for the same phantom conditions, demonstrating an over 2,000× improvement in SBR. In the current configuration, the fiber bundle system is able to complete a full measurement sequence capable of quantifying $\mu_{\mathrm{a}}, \mu_{\mathrm{s}}{ }^{\prime}, \gamma$, and $\mathrm{Q} \mu_{\mathrm{a}}^{\mathrm{f}}$ of a localized volume of tissue in less than eight seconds. With an overall bundle diameter slightly over $1.0 \mathrm{~mm}$, the bundle used in this study is capable of being delivered to hollow organs through an endoscope or used as a simple handheld probe for superficial tissues and open surgical sites. Additionally, the maximum effective fiber diameter of $1.0 \mathrm{~mm}$ provides measurement depth sufficient for probing the superficial vasculature lying beneath epithelial tissue and the longer photon path-length increases the sensitivity to low concentrations of tissue chromophores.

While the liquid optical phantoms used in this study are well-suited for validation and characterization of the system, they represent an idealized tissue environment in which the optical properties are spatially homogeneous. The different fiber diameters used in MDSFR measure over different tissue depths, but assume consistent scattering properties in the different volumes. Because the MDSFR system guarantees co-localization of the effective fiber diameter, the spatially averaged scattering properties sampled by each fiber diameter are expected to be quite similar in most tissues. However, the effect of layered tissue with stratified optical properties on MDSFR measurements has yet to be investigated. Similarly, the quantitative fluorescence model assumes homogeneous distribution of fluorophores as well as scatterers, and could be potentially confounded by unequal distributions of one or the other. Given the limited measurement 
volumes (4), any such heterogeneity is expected to be small. However, the effects of tissue optical property variations on the models will be the subject of future study.

\section{CONCLUSION}

We have demonstrated a robust MDSFR/SFF system capable of quantifying $\mu_{\mathrm{a}}, \mu_{\mathrm{s}}{ }^{\prime}, \gamma$, and $\mathrm{Q} \mu_{\mathrm{a}}^{\mathrm{f}}$ from a small volume of tissue in one eight-second measurement, making it well suited for use in a clinical environment. The increased speed and robustness in comparison to the previous pinhole-based proof-of-concept system are a result of the elimination of freespace optics. While system calibration is critical to accurate optical property measurement, the daily calibration of the system requires only two simple measurements in liquid samples and an automated integrating sphere measurement, which are guided by the user interface for easy use by clinicians.

Using liquid optical phantoms, we have demonstrated that the system uses a fiber bundle to accurately simulate a variable-diameter solid-core fiber for both SFR and SFF spectroscopy. The effective path-lengths and reflectances measured from the fiber bundle system match those measured by the solid-core fibers. Notably, we have used this system to demonstrate combined MDSFR/SFF spectroscopy, wherein the scattering and absorption properties are accurately quantified and then used to provide correction for quantitative fluorescence spectroscopy. Future work will investigate the use of this technique in stratified tissue optical properties and the integration of this system into clinical optical property measurements.

\section{REFERENCES}

[1] I. J. Bigio and S. G. Bown, "Spectroscopic Sensing of Cancer and Cancer Therapy: Current Status of Translational Research," Cancer Biology \& Therapy 3(3), 259-267 (2004)

[2] A. Wax and V. Backman, Biomedical Applications of Light Scattering, McGraw-Hill Professional Publishing (2009).

[3] J. R. Lakowicz, Principles of Fluorescence Spectroscopy, Springer London, Limited (2006).

[4] S. C. Kanick, D. J. Robinson, H. J. C. M. Sterenborg and A. Amelink, "Monte Carlo analysis of single fiber

reflectance spectroscopy: photon path length and sampling depth," Physics in Medicine and Biology 54(22), 6991 (2009)

[5] S. C. Kanick, H. J. C. M. Sterenborg and A. Amelink, "Empirical model of the photon path length for a single fiber reflectance spectroscopy device," Opt. Express 17(2), 860-871 (2009)

[6] S. C. Kanick, U. A. Gamm, M. Schouten, H. J. C. M. Sterenborg, D. J. Robinson and A. Amelink, "Measurement of the reduced scattering coefficient of turbid media using single fiber reflectance spectroscopy: fiber diameter and phase function dependence," Biomed. Opt. Express 2(6), 1687-1702 (2011)

[7] T. J. Pfefer, D. Y. Paithankar, J. M. Poneros, K. T. Schomacker and N. S. Nishioka, "Temporally and spectrally resolved fluorescence spectroscopy for the detection of high grade dysplasia in Barrett's esophagus," Lasers in Surgery and Medicine 32(1), 10-16 (2003)

[8] J. C. Finlay, T. C. Zhu, A. Dimofte, D. Stripp, S. B. Malkowicz, T. M. Busch and S. M. Hahn, "Interstitial Fluorescence Spectroscopy in the Human Prostate During Motexafin Lutetium-Mediated Photodynamic Therapy," Photochemistry and Photobiology 82(5), 1270-1278 (2006)

[9] S. C. Kanick, C. van der Leest, J. G. J. V. Aerts, H. C. Hoogsteden, S. Kaščáková, H. J. C. M. Sterenborg and A. Amelink, "Integration of single-fiber reflectance spectroscopy into ultrasound-guided endoscopic lung cancer staging of mediastinal lymph nodes," Journal of Biomedical Optics 15(1), 017004-017004 (2010)

[10] S. C. Kanick, C. van der Leest, R. S. Djamin, A. M. Janssens, H. C. Hoogsteden, H. J. C. M. Sterenborg, A. Amelink and J. G. J. V. Aerts, "Characterization of Mediastinal Lymph Node Physiology In Vivo by Optical Spectroscopy during Endoscopic Ultrasound-Guided Fine Needle Aspiration," Journal of Thoracic Oncology 5(7), 981$987(2010)$

[11] S. C. Kanick, D. J. Robinson, H. J. C. M. Sterenborg and A. Amelink, "Method to quantitate absorption coefficients from single fiber reflectance spectra without knowledge of the scattering properties," Opt. Lett. 36(15), 2791-2793

(2011)

[12] U. A. Gamm, S. C. Kanick, H. J. C. M. Sterenborg, D. J. Robinson and A. Amelink, "Measurement of tissue scattering properties using multi-diameter single fiber reflectance spectroscopy: in silico sensitivity analysis," Biomed.

Opt. Express 2(11), 3150-3166 (2011) 
[13] U. A. Gamm, S. C. Kanick, H. J. C. M. Sterenborg, D. J. Robinson and A. Amelink, "Quantification of the reduced scattering coefficient and phase-function-dependent parameter of turbid media using multidiameter single fiber reflectance spectroscopy: experimental validation," Opt. Lett. 37(11), 1838-1840 (2012)

[14] A. Ishimaru, Wave propagation and scattering in random media, Academic Press, New York (1978).

[15] V. Turzhitsky, A. Radosevich, J. D. Rogers, A. Taflove and V. Backman, "A predictive model of backscattering at subdiffusion length scales," Biomedical optics express 1(3), 1034-1046 (2010)

[16] S. C. Kanick, U. A. Gamm, H. J. C. M. Sterenborg, D. J. Robinson and A. Amelink, "Method to quantitatively estimate wavelength-dependent scattering properties from multidiameter single fiber reflectance spectra measured in a turbid medium," Opt. Lett. 36(15), 2997-2999 (2011)

[17] F. van Leeuwen-van Zaane, U. A. Gamm, P. B. A. A. van Driel, T. J. A. Snoeks, H. S. de Bruijn, A. van der Ploegvan den Heuvel, I. M. Mol, C. W. G. M. Löwik, H. J. C. M. Sterenborg, A. Amelink and D. J. Robinson, "In vivo quantification of the scattering properties of tissue using multi-diameter single fiber reflectance spectroscopy," Biomed. Opt. Express 4(5), 696-708 (2013)

[18] S. C. Kanick, D. J. Robinson, H. J. C. M. Sterenborg and A. Amelink, "Semi-empirical model of the effect of scattering on single fiber fluorescence intensity measured on a turbid medium," Biomed. Opt. Express 3(1), 137-152 (2011)

[19] C. L. Hoy, U. A. Gamm, H. J. C. M. Sterenborg, D. J. Robinson and A. Amelink, "Use of a coherent fiber bundle for multi-diameter single fiber reflectance spectroscopy," Biomed. Opt. Express 3(10), 2452-2464 (2012)

[20] R. Sjöback, J. Nygren and M. Kubista, "Absorption and fluorescence properties of fluorescein," Spectrochimica Acta Part A: Molecular and Biomolecular Spectroscopy 51(6), L7-L21 (1995) 